\title{
MÉTOdO DE ELEMENTOS FINITOS PARA CARACTERIZAR ANTENAS MICROSTRIP CON DIFERENTE SUBSTRATO PARA SENSORES DE ALTA TEMPERATURA
}

\author{
Finite ELEMENT METHOD TO CHARACTERIZE MICROSTRIP \\ ANTENNAS WITH DIFFERENT SUBSTRATE FOR HIGH \\ TEMPERATURE SENSORS
}

\section{Leonardo Andres Perez ${ }^{1}$, Diego Fernando Sendoya ${ }^{2}$, Carlos Alberto Vera ${ }^{3}$}

Escuela de Ciencias Básicas Tecnología e Ingeniería, Universidad Nacional Abierta y a Distancia-UNAD

Recibido: 07/04/2017• Aprobado: 25/05/2017

\section{RESUMEN}

La integración de la antena microstrip al sensor SAW representa una atractiva solución para monitorear altas temperaturas a distancia. En aplicaciones donde se utilizan hornos industriales es indispensable supervisar la temperatura a través de sensores operados con tecnología infrarroja o inalámbrica. El propósito de éste estudio fue caracterizar el patrón de radiación de una antena microstrip hecha sobre el mismo substrato piezoeléctrico que forma el sensor SAW, con el fin de desarrollar un medidor de altas temperaturas interrogado inalámbricamente. Este estudio hace parte de la etapa de diseño de una antena para el SAW, la cual será fabricada posteriormente. A través del método de elementos finitos, tres antenas microstrip hechas con substratos de Quartz, Langasite y Dilithium Tetraborate fueron modeladas y simuladas. La simulación permitió establecer parámetros tales como comportamiento de los planos E-field (eléctrico) y H-field (magnético), dirección de la máxima radiación, ancho del lóbulo principal y la directividad. Los resultados mostraron que la antena hecha con Quartz tiene una potencia máxima de radiación de $2 \mathrm{~dB}$, con un ancho del lóbulo principal de $48^{\circ}$. Para la antena de Langasite, se obtuvo una potencia de radiación de $-12 \mathrm{~dB}$, con un ancho del lóbulo de $30^{\circ}$. Y para la antena de Dilithium Tetraborate, la potencia de radiación fue de $-3.2 \mathrm{~dB}$, con un ángulo de radiación de $78^{\circ}$.

Palabras clave: antena microstrip, elementos finitos, sensor SAW, substrato piezoeléctrico, temperatura.

\section{Abstract}

The integration of a microstrip antenna into a SAW sensor represents an attractive solution to monitor high temperature remotely. In applications where industrial furnaces are used, it is essential to monitor the temperature through

\footnotetext{
1 leonardo.perez@unad.edu.co, http://orcid.org/0000-0002-0636-0870

2 diego.sendoya@usco.edu.co, https://orcid.org/0000-0001-6197-935

3 carlos.vera@unad.edu.co, https://orcid.org/0000-0002-3315-858X
} 
sensors operated by infrared or wireless technology. The purpose of this study was to characterize the radiation pattern of a microstrip antenna composed of the same piezoelectric substrate as the SAW sensor, in order to develop a wirelessly-interrogated high temperature sensor. This study is part of the design of an antenna for a SAW device, which is in process to be manufactured. By means of Finite Element Method (FEM), three microstrip antennas made of substrates from Quartz, Langasite and Dilithium Tetraborate were modeled and simulated. The simulation allowed to establish parameters such as the behavior of E-field (electrical) and H-field (magnetic) planes, direction of the maximum radiation, width of the main lobe and directivity. The results showed that the antenna of Quartz exhibited a maximum radiation power of $2 d B$, with a main lobe width of $48^{\circ}$. For the Langasite antenna, a radiation power of $-12 \mathrm{~dB}$ was obtained, with a lobe width of $30^{\circ}$. And for the Dilithium Tetraborate antenna, the radiation power was $-3.2 \mathrm{~dB}$, with a radiation angle of $78^{\circ}$.

Key words: finite element, microstriop antenna, piezoelectric substrate, SAW sensor, temperature.

\section{INTRODUCCIÓN}

Los sensores juegan un papel fundamental en el desarrollo de la cuarta revolución industrial, en donde máquinas inteligentes se conectan e interactúan en procesos industriales dinámicos (Rossi, 2017). Para una buena conectividad es necesario una apropiada infraestructura del Internet de las cosas, compuesta por sensores capaces de monitorear en tiempo real múltiples variables físico-químicas. Hoy en día existen sensores para diversas aplicaciones, tales como monitoreo de la temperatura, presión, humedad, $\mathrm{PH}$, entre otros. Como el mercado de éstos dispositivos ha venido creciendo, pasando de 86.4 billones de dólares en 2014 a 95.3 millones en 2015 (Rix, 2015), se espera que en el 2020 surjan nuevos sensores capaces de medir magnitudes más complejas y con interrogación inalámbrica.

Esfuerzos sustanciales han sido llevados a cabo para demostrar la funcionalidad de los sensores de onda superficial, en inglés Surface Acoustic Wave (SAW). Estos dispositivos tienen la ventaja de ser pequeños y tienen múltiples aplicaciones. Dentro de éstas, PérezCortes et al. (2016) y Pérez-Cortes (2017) demostró que la integración del nano material paladio-plata sobre la superficie del SAW puede detectar concentraciones de hidrógeno en el aire, a partir de

2000 ppm. Fu et al. (2017) desarrolló un sensor SAW capaz de medir temperaturas desde $0^{\circ} \mathrm{C}$ a $130^{\circ} \mathrm{C}$, por su parte Rimeika et al. (2009) demostró que una capa de hematoporphyrin depositada sobre un dispositivo SAW puede medir la humedad en un rango de $13 \%$ a 33\%, en otro caso, Rana et al. (2017) integró una capa de $\mathrm{ZnO}$ sobre la superficie de un SAW resonador para medir concentraciones de dióxido de nitrógeno, a partir de $400 \mathrm{ppb}$; todas estas investigaciones muestran la capacidad que tiene la tecnología SAW para medir diversas variables físico-químicas.

Una extensión de la tecnología SAW es su adaptación para ser interrogada a distancia. Para ello, una antena debe ser integrada a la estructura SAW y un transceptor establece la comunicación. Kim et al. (2015) demostró que un sensor SAW de temperatura conectado a una antena dipolo, hecha sobre un circuito impreso, es capaz de medir la temperatura del concreto en sus diferentes fases. Wang et al. (2014) desarrolló un sensor SAW inalámbrico para medir las concentraciones de dióxido de carbono en el aire, en donde integró antenas comerciales de frecuencia central de 6.1 MHz. Tang et al. (2015) estudió un sistema de medición de presión del agua utilizando un sensor SAW de presión, el cual integró dos antenas tipo dipolo helicoidal operando a una frecuencia de $433 \mathrm{MHz}$. Estos y otros estudios evidencian la funcionalidad del sensor SAW para la interrogación a distancia. 
Para mediciones a altas temperaturas, los sensores SAW inalámbricos presentan retos importantes. Los materiales utilizados en la estructura del sensor deben soportar altas temperaturas para evitar la dilatación térmica o fracturación. Tortissier et al. (2009) demostró la funcionalidad de un sensor SAW para medir temperaturas tan altas como $450^{\circ} \mathrm{C}$, haciendo uso del material Langasite como substrato piezoeléctrico. Este material tiene la ventaja de no fracturarse a elevadas temperaturas. Además del material del SAW, es importante seleccionar el substrato apropiado para la antena con el fin de evitar fallas por temperatura. En ese aspecto, Fachberger et al. (2009) desarrolló un sistema de monitoreo inalámbrico basado en SAW para medir la temperatura dentro de una línea de producción metalúrgica. El sistema funcionó adecuadamente pero su temperatura máxima fue limitada por la temperatura que soporta la antena del sensor, es decir, $60^{\circ} \mathrm{C}$. Por consiguiente, se tienen grandes retos en la selección de los materiales adecuados para la antena.

Este artículo presenta el estudio de una antena microstrip que puede soportar altas temperaturas. Como el substrato de ésta antena es el mismo substrato piezoeléctrico usado en el sensor SAW, la antena puede ser fabricada con materiales que soportan elevadas temperaturas, tales como Langasite. Este estudio se realizó a través del método de elementos finitos para tres tipos de substratos piezoeléctricos: Quartz, Langasite y Dilithium Tetraborate. Los resultados del estudio describen las características de radiación de las antenas, involucrando su comportamiento de los planos E-field (eléctrico) y H-field (magnético), dirección de la máxima radiación, entre otros. Este modelamiento hace parte del diseño de la antena del SAW, la cual será desarrollada posteriormente.

La estructura de éste documento se organiza en 4 secciones además de esta introducción: la sección II describe los fundamentos de la antena microstrip y el sensor SAW. La sección III describe el modelamiento de la antena por el método de elementos finitos. La sección IV muestra los resultados y discusiones de las simulaciones. Finalmente, la sección $\mathrm{V}$ presenta las conclusiones y perspectivas de ésta investigación.

\section{ANTENA MICROSTRIP Y SAW}

La antena microstrip es una tecnología RF que se fabrica con técnicas de circuito impreso. La Figura 1 muestra la estructura de esta antena, compuesta por tres elementos: 1) el área superior impresa, 2) el dieléctrico o substrato, y 3) el plano de masa (en inglés, ground plane). El área superior se divide en dos secciones: 1) la línea de alimentación (en inglés, feed line) y el parche (en inglés, patch). El patch tiene múltiples configuraciones, tales como un dipolo, cuadrado, rectangular, pentagonal, triangular y circular.

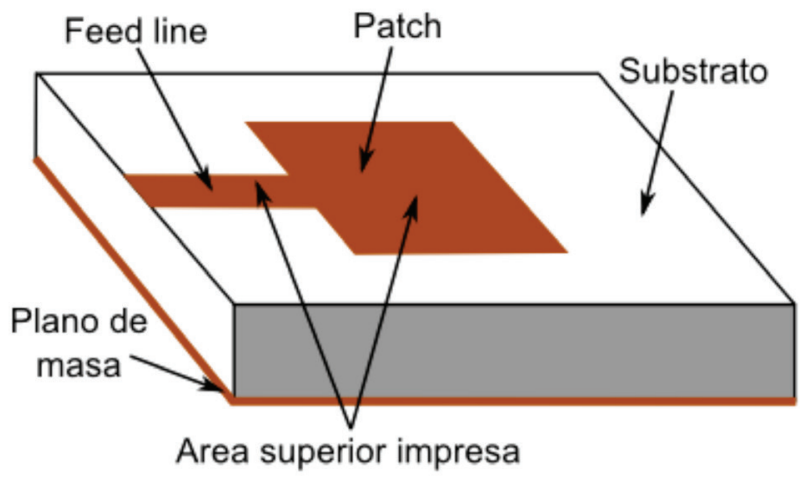

Fig. 1. Esquema de una antena microstrip. Fuente: autores

En este trabajo, la antena microstrip es estudiada integrándola al sensor SAW. Un sensor SAW resonador está formado por un electrodo metálico de tipo peine-interdigital (denominado en inglés, InterDigital Transducer - IDT) y una estructura conocida como reflector, ambos depositados sobre la superficie de un substrato piezoeléctrico. Este dispositivo opera induciendo ondas mecánicas (o acústicas) que se propagan sobre la superficie del substrato. Durante la propagación, el reflector recibe estas ondas y las retorna al IDT generando una señal eléctrica a la salida. Como estas ondas son sensibles a múltiples parámetros físico-químicos (temperatura, presión, humedad, etc.), la estructura del SAW puede ser adaptada para ser un sensor. La integración de una antena microstrip al IDT, sobre el mismo sustrato piezoeléctrico del SAW, permitiría irradiar la señal eléctrica de salida y crear un sensor con transmisión inalámbrica. Figura 2. 


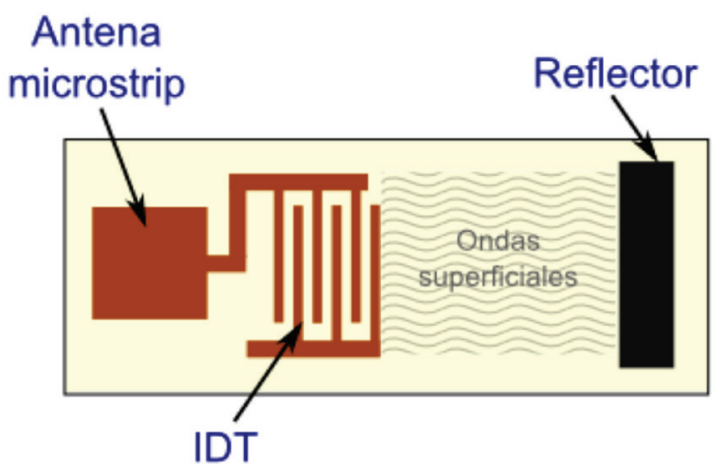

Fig. 2. Vista superior de una antena microstrip integrada al sensor SAW resonador. Fuente: autores

En éste estudio, la antena microstrip se modela por el método de elementos finitos, a través del software COMSOL Multiphysics. El modelamiento caracteriza el comportamiento de la antena utilizando tres diferentes substratos piezoeléctricos: Quartz, Langasite y Dilithium tetraborate. Los aspectos del modelamiento y simulación de la antena son descritos a continuación.

\section{Modelamiento de la antena}

La Figura 3 muestra el modelo de la antena con su mallado. En el modelamiento se estableció que el feed line, el patch y el ground plane son conductores perfectos, y la trasmisión de la señal desde el exterior hasta el feed line es representada como un circuito eléctrico en serie, de la misma forma como se representa una línea de transmisión en telecomunicaciones.

Este modelo consideró una condición de frontera con el fin de que las ondas electromagnéticas se propaguen sin ninguna reflexión dentro de una región delimitada. En el software, ésta región tiene forma esférica con radio igual a la longitud del substrato. En la discretización fue definido con un mallado tetrahedral no uniforme, con dimensiones entre 18 $\mathrm{mm}$ y $3.24 \mathrm{~mm}$.

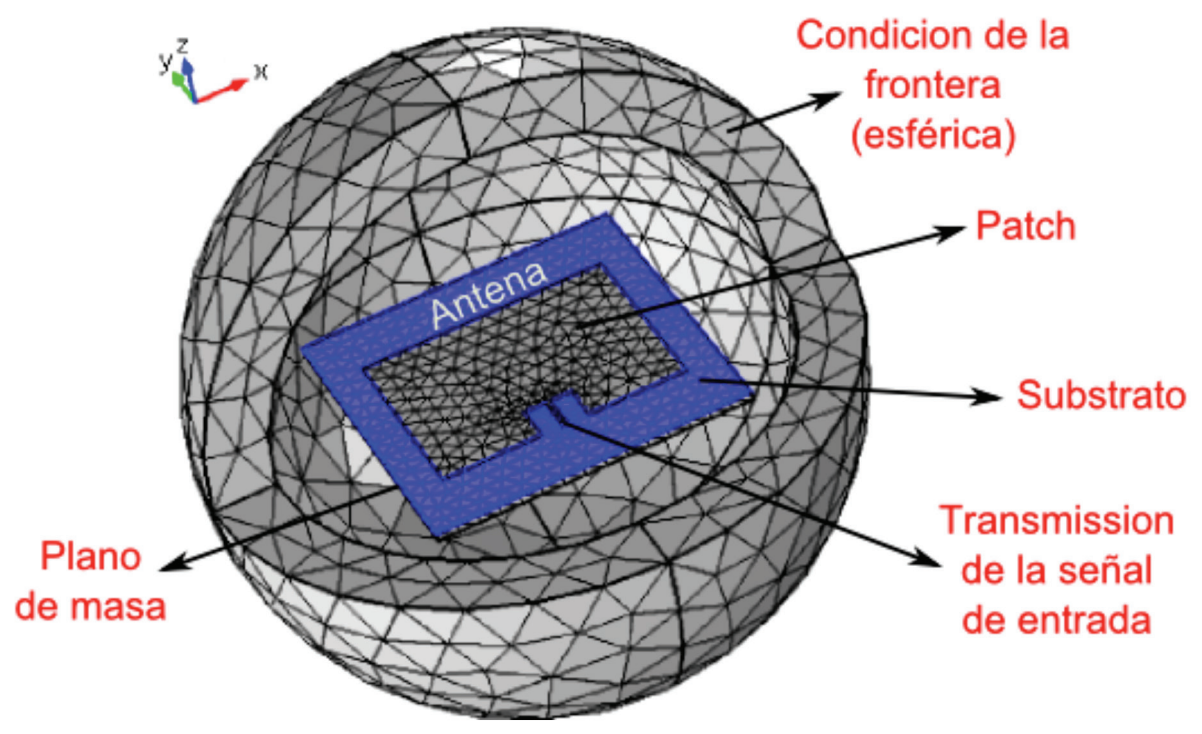

Fig. 3. Modelamiento de la antena microstrip por el método de elementos finitos.

Fuente: autores 


\subsection{Dimensionamiento de la antena}

Para caracterizar la antena usando el modelo, fueron ingresadas las dimensiones del patch, el feed line y el plano de masa. Para lograr que la antena irradie la energía eficiente, el patch debe dimensionarse siguiendo las siguientes ecuaciones (Ionescu, 2017):

$$
W_{p}=\frac{c}{2 f_{r} \sqrt{\frac{\varepsilon_{r}+1}{2}}}
$$

Donde $W_{p}$ es el ancho del patch, $c$ es la velocidad de las ondas electromagnéticas en el espacio libre $\left(3 \cdot 10^{8}\right.$ $\left.\mathrm{m}^{-1} . \mathrm{s}\right)$, fr es la frecuencia de resonancia de la antena $(2.45 \mathrm{GHz})$ y $\varepsilon r$ es la permitividad relativa del substrato. La longitud de patch $L_{p}$ se obtiene por:

$$
L_{p}=\frac{c}{2 f_{r} \sqrt{\varepsilon_{f}}}-2 \Delta L
$$

Donde $\Delta \mathrm{L}$ y ef son:

$$
\begin{gathered}
\Delta L=0.412 \cdot h \cdot \frac{\left(\varepsilon_{f}+0.3\right)}{\left(\varepsilon_{f}-0.258\right)} \cdot \frac{\left(\frac{W_{p}}{h}+0.264\right)}{\left(\varepsilon_{f}+0.8\right)} \\
\varepsilon_{f}=\frac{\varepsilon_{r}+1}{2}+\frac{\varepsilon_{r}+1}{2}\left[1+12 \frac{h}{W_{p}}\right]^{-1 / 2}
\end{gathered}
$$

Siendo $\varepsilon f$ es la permitividad eficiente y $h$ es la altura (o grosor) del substrato. Las dimensiones mínimas del substrato (longitud $L_{s}$ y ancho $W$ ) se obtienen por:

$$
\begin{gathered}
L_{s}=6 \cdot h+L \\
W_{s}=6 \cdot h+W
\end{gathered}
$$

El ancho $W_{f t}$ y longitud $L_{f}$ del feed line se derivan de las siguientes ecuaciones:

$$
\begin{gathered}
W_{f l}=\frac{2 h}{\pi \cdot\left(B-1-m+\left(\frac{\varepsilon_{r}-1}{2 \varepsilon_{r}}\right) \cdot\left(n+\frac{0.39 \cdot 0.61}{\varepsilon_{r}}\right)\right.} \\
L_{f l}=\frac{L_{p}}{\pi \cdot \cos ^{-1}\left(\sqrt{\frac{Z_{\text {in }}}{r_{\text {in }}}}\right)}
\end{gathered}
$$

Donde:

$$
\begin{gathered}
B=\frac{60 \pi^{2}}{Z_{\text {in }} \sqrt{\varepsilon_{r}}} \\
m=\ln (2 \cdot B-1) \\
n=\ln (B-1) \\
r_{\text {in }}=\frac{1}{2\left(g_{1}+g_{12}\right)}
\end{gathered}
$$

Siendo $\mathrm{Z}_{\text {in }}$ la impedancia de entrada de la antena (típicamente $50 \Omega$ ), y $g_{1}, g_{12}$ son admitancias asociadas

\begin{tabular}{|c|c|c|c|}
\hline & Quartz & Langasite & $\begin{array}{l}\text { Dilithium } \\
\text { tetraborate }\end{array}$ \\
\hline $\begin{array}{c}\text { Grosor del } \\
\text { substrato }(b)\end{array}$ & $1.6 \mathrm{~mm}$ & $1.6 \mathrm{~mm}$ & $1.6 \mathrm{~mm}$ \\
\hline $\begin{array}{l}\text { Ancho del } \\
\text { Patch }\left(W_{P}\right)\end{array}$ & $35.58 \mathrm{~mm}$ & $16.36 \mathrm{~mm}$ & $28.65 \mathrm{~mm}$ \\
\hline $\begin{array}{l}\text { Longitud del } \\
\text { patch }\left(L_{p}\right)\end{array}$ & $27.27 \mathrm{~mm}$ & $11.53 \mathrm{~mm}$ & $21.24 \mathrm{~mm}$ \\
\hline $\begin{array}{l}\text { Ancho del feed } \\
\text { line }\left(W_{f 1}\right)\end{array}$ & $2.72 \mathrm{~mm}$ & $0.1352 \mathrm{~mm}$ & $1.71 \mathrm{~mm}$ \\
\hline $\begin{array}{l}\text { Longitud del } \\
\text { feed line }\left(L_{f 1}\right)\end{array}$ & $10.23 \mathrm{~mm}$ & $5.04 \mathrm{~mm}$ & $8.39 \mathrm{~mm}$ \\
\hline $\begin{array}{l}\text { Ancho del subs- } \\
\text { trato }\left(W_{s}\right)\end{array}$ & $46 \mathrm{~mm}$ & $27 \mathrm{~mm}$ & $39 \mathrm{~mm}$ \\
\hline $\begin{array}{l}\text { Longitud del } \\
\text { substrato }\left(L_{s}\right)\end{array}$ & $46 \mathrm{~mm}$ & $27 \mathrm{~mm}$ & $39 \mathrm{~mm}$ \\
\hline $\begin{array}{l}\text { Permitividad } \\
\text { relativa }\left(\varepsilon_{r}\right)\end{array}$ & 4.92 & 28 & 8.13 \\
\hline
\end{tabular}
al patch. La tabla I muestra las dimensiones obtenidas por las ecuaciones anteriores para los substratos de Quartz, Langasite y Dilithium Tetraborate.

El modelamiento y posterior simulación fue llevado a cabo para cada uno de los materiales y dimensiones indicadas en la Tabla 1. Basado en esta información, los resultados de las simulaciones son presentadas a continuación.

\section{TABLA 1.}

Dimensiones de la antena microstrip para los substratos Quartz, Langasite y Dilithium tetraborate

Fuente: Gualtieri, 1994. 


\section{Resultados y Discusiones}

La Figura 4A-B-C muestra en 3D el patrón de radiación normalizado (far field normal) en decibeles de las antenas con substrato de Quartz, Langasite y Dilithium Tetraborate, respectivamente. Además, se incluye el diagrama polar con sus componentes del plano E-field (eléctrico) y H-field (magnético). El E-field se orienta $90^{\circ}$ en el plano polar, y el H-field se orienta a $180^{\circ}$ en éste mismo plano. Para la antena de Quartz, se observa que el patrón contiene un lóbulo principal orientado hacia el eje $+\mathrm{z}$ (es decir, la antena tiene directividad hacia $+z$ ), y un lóbulo secundario hacia el eje -z. El valor máximo del lóbulo principal se aproxima a 2 $\mathrm{dB}$, y para el lóbulo secundario es de $-8 \mathrm{~dB}$. El ancho del lóbulo principal, definido como el ángulo que forma el patrón de radiación en el punto $-3 \mathrm{~dB}$ de su valor máximo, es de $48^{\circ}$. Para la antena de
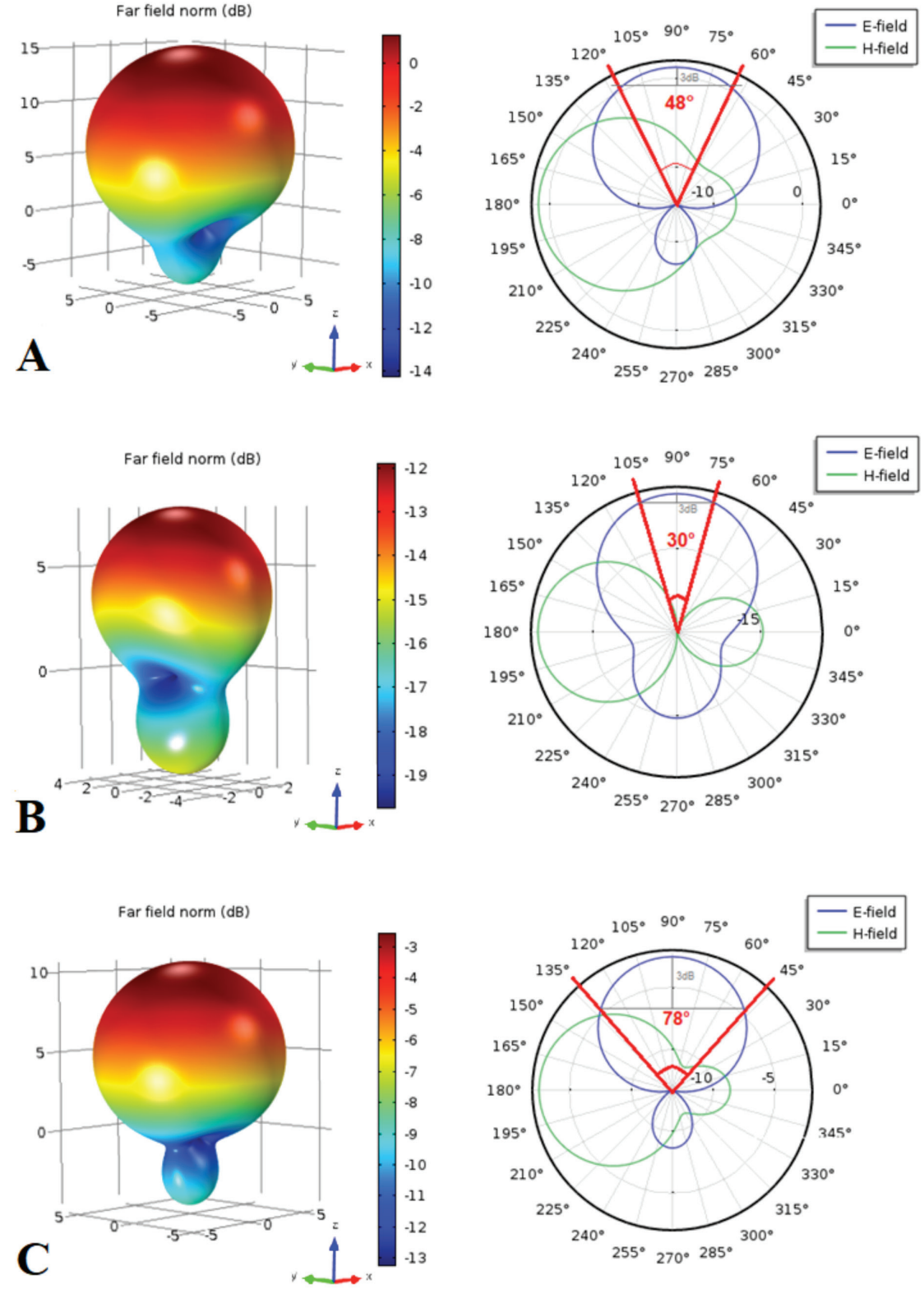

Fig. 4. Resultados de la simulación de la antena. A) Antena con substrato de Quartz, B) antena con substrato de Langasite, y C) antena con substrato de Dilithium Tetraborate.

Fuente: autores 
Langasite, su lóbulo principal también se orienta a $+z$, y su lóbulo secundario hacia $-z$. El valor máximo del lóbulo principal está en $-12 \mathrm{~dB}$, y el lóbulo secundario es de $-15 \mathrm{~dB}$. El ancho del lóbulo principal es de $30^{\circ}$. Y para la antena de Dilithium Tetraborate, su lóbulo principal tiene dirección hacia $+z$, con un lóbulo secundario hacia $-z$. El valor máximo del lóbulo principal se aproxima a $-3.2 \mathrm{~dB}$, y el lóbulo secundario es de $-8.5 \mathrm{~dB}$, con un ancho del lóbulo principal de $78^{\circ}$. Las distintas permitividades de cada una de las antenas conlleva a estos comportamientos diversos.

La Tabla 2 compara las características de radiación obtenidas, a fin de determinar el cristal adecuado para la antena. Se observa que las tres antenas irradian su máxima potencia hacia la dirección $+z$. En términos de potencia normal del lóbulo principal, la antena con substrato de Quartz exhibió la máxima radiación. En contraste, la antena de Langasite mostró la más baja potencia. Si se compara el ancho del lóbulo principal, la antena de Langasite exhibió el ángulo más corto lo que significa que ésta antena tiene una alta directividad. Por consiguiente, si se considera que el dispositivo SAW requiere un gran alcance en todas las direcciones, el cristal Quartz es el más apropiado para la antena. En caso que se requiera una mayor directividad sin importar el alcance, el cristal Langasite es el adecuado. Si se selecciona el Quartz, es importante realizar estudios para definir si este material es capaz de soportar altas temperaturas.

\section{Tabla 2.}

Resultados de las características de radiación de las antenas de quartz, langasite y dilitrhium tetraborate

\begin{tabular}{|c|c|c|c|}
\hline & Quartz & Langasite & $\begin{array}{c}\text { Dilithium } \\
\text { tetraborate }\end{array}$ \\
\hline $\begin{array}{c}\text { Direction } \\
\text { de radiación }\end{array}$ & $+Z$ & $+Z$ & $+Z$ \\
\hline $\begin{array}{c}\text { Potencia normal } \\
\text { del lóbulo } \\
\text { principal }\end{array}$ & $2 \mathrm{DB}$ & $-12 \mathrm{DB}$ & $3.2 \mathrm{DB}$ \\
\hline $\begin{array}{c}\text { Ancho del lobulo } \\
\text { principal }\end{array}$ & $48^{\circ}$ & $30^{\circ}$ & $78^{\circ}$ \\
\hline
\end{tabular}

Fuente: autores

\section{Conclusiones}

El estudio, por el método de elementos finitos, determinó las características de radiación de antenas microstrips hechas con substrato de Quartz, Langasite y Dilithium Tetraborate. Los resultados muestran que las tres antenas irradian su máxima potencia hacia el eje $+z$. El material Langasite, el cual ha sido reportado como capaz de resistir altas temperaturas, exhibió el menor ángulo del lóbulo principal y una máxima potencia de radiación de $-12 \mathrm{~dB}$. Por estas características, este material puede ser utilizado para la antena del SAW exhibiendo un regular alcance de transmisión y buena directividad. Con los otros materiales también es posible fabricar la antena microstrip, pero se requieren futuros estudios que indiquen su capacidad para soportar altas temperaturas.

Como futuro trabajo, el modelo de antena microstrip con materiales piezoeléctricos será validado con experimentos. Esta validación involucraría el desarrollo de las antenas y su posterior caracterización del patrón de radiación. La validación permitiría dar solución a la problemática de fabricar una antena que soporte altas temperaturas para ser integradas a los sensores SAW.

\section{REFERENCIAS}

Fu, C., Ke, Y., Li, M., Luo, J., Li, H., Liang, G. \& Fan, P. (2017). Design and Implementation of $2.45 \mathrm{GHz}$ Passive SAW Temperature Sensors with BPSK Coded RFID Configuration, Sensors, $17(8), 1849$.

Fachberger, R. \& Erlacher, A. (2009). Monitoring of the temperature inside a lining of a metallurgical vessel using a SAW temperature sensor. Procedia Chemistry, 1(1), 1239-1242.

Gualtieri, J. G., Kosisnski, J. A. \& Ballato, A. (1994). Piezoelectric materials for acoustic wave applications. IEEE transaction of ultrasonics, 41(1), 53-59.

Ionescu V. (2017). Numerical modelling of a microstrip patch antenna for wireless applications, 10th International symposium on advanced topics in electrical engineering, p. 340-435.

Kim, J., Rodriguez, L., Smith, S., Figueroa, J. A., Malocha, D. \& Nam, B. H. (2015). Concrete temperature monitoring using passive wireless surface acoustic wave sensor system, Sensors and Actuators A: Physical, 224(1), 131-139. 
Perez-Cortes, L. (2017). Conception and realization of a SAW sensor based on a nanostructured-catalytic material for the detection of carbon monoxide and hydrogen, Tesis de dcotorado, Grenoble: Université Grenoble Alpes.

Perez-Cortes, L., Hernandez, C., Mazingue, T. \& Lomello-Tafin, M., (2016). Functionality of Surface Acoustic Wave (SAW) transducer for palladium-platinum-based hydrogen sensor. Sensors and Actuators A: Physical, 251, 35-41.

Rana, L., Gupta, R., Tomar, M. \& Gupta V. (2017) ZnO/STQuartz SAW resonator: An efficient NO2 gas sensor. Sensors and Actuators B: Chemical, 252, 840-845.

Rimeika, R., Ciplis, D., Poderys, V. Rotomskis, R. \& Shur, M.S. (2009). Fast-response surface acoustic wave humidity sensor based on hematoporphyrin film. Sensors and Actuators B: Chemical., 137(2), 592-596.

Rix, N. (2015). The current state of the sensors community and technology in an increasingly sensed world, strategy and funding opportunities, Knowledge Transfer Network (KTN-UK). Recuperado en: https://www.southampton.ac.uk/assets/imported/ transforms/content-block/UsefulDownloads_Download/4C25 C0D86ABA42E593525F90CA12891C/Nigel\%20Rix.pdf
Rossi, B. (2017). The fourth industrial revolution: technology alliances lead the charge. Recuperado en: https://www.information-age.com/fourth-industrial-revolution-technology-alliances-lead-charge-123465633/

Tang, Z., Wu, W. \& Gao, J. (2015). Water pressure sensing based on wireless passive SAW technology, Int. conference on computing and control for the water industry. Procedia Engineering, $119,892-900$.

Tortissier, G., Blanc, L., Tetelin, A., Lachaud, J. A., Benoit, M., Conédéra, V., Dejous, C., Rebière, D. (2011). Langasite based surface acoustic wave sensors for high temperature chemical detection in harsh environment: Design of the transducers and packaging. Sensors and Actuators B: Chemical, 156(2), 510-516.

Wang, Y., Chyu, M. K. \& Wang, Q.M. (2014). Passive wireless surface acoustic wave $\mathrm{CO} 2$ sensor with carbon nanotube nanocomposite as an interface layer, Sensors and Actuators A: Physical, 220(1), p. 34-44. 


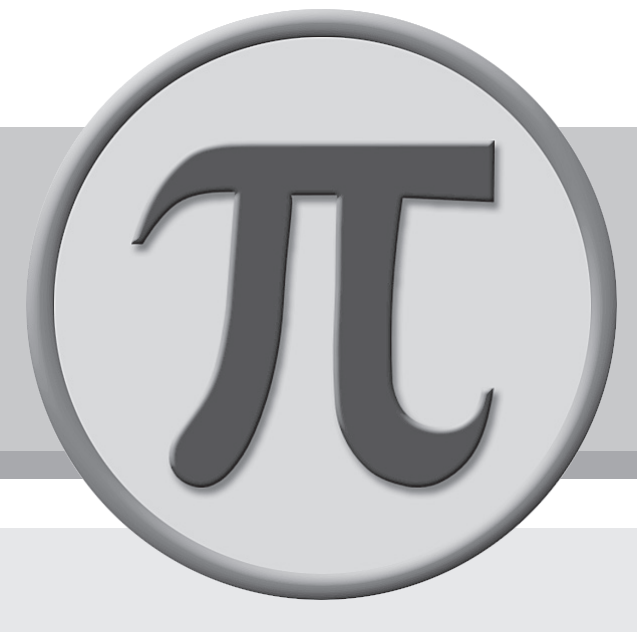


